\title{
STABILITY ANALYSIS OF SEIVHR EPIDEMIC MODEL WITH SATURATED INCIDENCE RATE
}

\author{
Muhammad Altaf Khan ${ }^{\mathrm{a}}$, Sehra ${ }^{\mathrm{a}}$, Saeed Islam ${ }^{\mathrm{a}}$, Sher Afzal Khan ${ }^{\mathrm{b}}$ \\ Department of Mathematics, Abdul Wali Khan University Mardan, Khyber \\ Pakhtunkhwa, Pakistan. \\ Department of Computer Sciences, Abdul Wali Khan University Mardan, Khyber \\ Pakhtunkhwa Pakistan.
}

\begin{abstract}
The aim of this paper, to analyze stability analysis of SEIVHR epidemic model with generalized non-linear incidence rate that spread in the host population horizontally. First, we formulate the model and find its basic reproduction number. Two equilibrium exists, namely; the disease free and endemic equilibrium. The disease free equilibrium is stable both locally and globally when the threshold quantity less than unity. The endemic equilibrium is locally and globally asymptotically stable when the threshold exceeds unity. Finally, we show some numerical results for the proposed model.
\end{abstract}

Key words: Epidemic model, Reproduction number, vaccination, local and global stability, numerical solution.

1. Introduction: Mathematical modeling plays an important role to understand the epidemiology of an infectious disease. These models, not only provides the qualitative behavior but also the complicated nonlinear process, about how to control the spread of such infectious disease [1,2]. Many mathematical models have been designed in the literature to understand the epidemiology of an infectious disease [3, 4, 5]. In these models the dynamical behavior and their qualitative study have been investigated. Many mathematical models have been formulated with difference incidence terms; i. e., the non-linear saturated incidence rate, bilinear incidence rate, generalized incidence rate etc. see for examples $[6,7,8,9]$.

Vaccination considered an important tool for the disease control. It is the administration of antigenic material that can stimulate an individual's immune system to produce the adaptive immunity to a pathogen. Its prevent morbidity from infection. Its effectiveness has been studied and verified widely; for example, the influenza vaccine, [10], the HPV vaccine [11] and the chicken pox vaccine [12]. According to the report of World Health Organization (WHO) vaccines are presently available to prevent, or to contribute control or prevention of, 25 vaccine-preventable infections.

In this paper, we consider an epidemic model of an SEIVHR with saturated incidence rate. First, we formulate the problem and the parameters therein. The model exhibits two type of equilibria; 1) disease free 2) endemic. A reproduction number for the model obtain, to investigate the stability of disease free and endemic equilibrium. The disease free equilibrium is stable locally and globally when the basic reproduction less than 1 . An unstable equilibrium exists, when this threshold parameter exceeds unity. In case, the threshold quantity exceeds unity the existence of the global stability of endemic equilibrium occur. Numerical results demonstrate the theoretical results.

The structure of the paper follows is as: The basic model construction and basic properties of the mode are discussed in section 2. The local stability of disease free and endemic equilibrium is discussed in section 3. In section 4 , the global stability of disease free and endemic equilibrium is presented. Some numerical results and discussion presented in section 5 . 
2. Mathematical formulation: In the given section, we formulate the epidemic SEIVHR epidemic model with nonlinear saturated incidence rate. We divide the total population into six sub classes i.e., Susceptible $S(t)$, the exposed individuals $\mathrm{E}(\mathrm{t})$, infected $\mathrm{I}(\mathrm{t})$, those who vaccinated by $\mathrm{V}(\mathrm{t})$, the hospitalized by $\mathrm{H}(\mathrm{t})$, and recovered by $\mathrm{R}(\mathrm{t})$. The total population size is denoted by $\mathrm{N}(\mathrm{t})$. We assume that the total population is constant. All the new born babies and migrated people are assumed to be susceptible.

The system that governs the differential equation is given by:

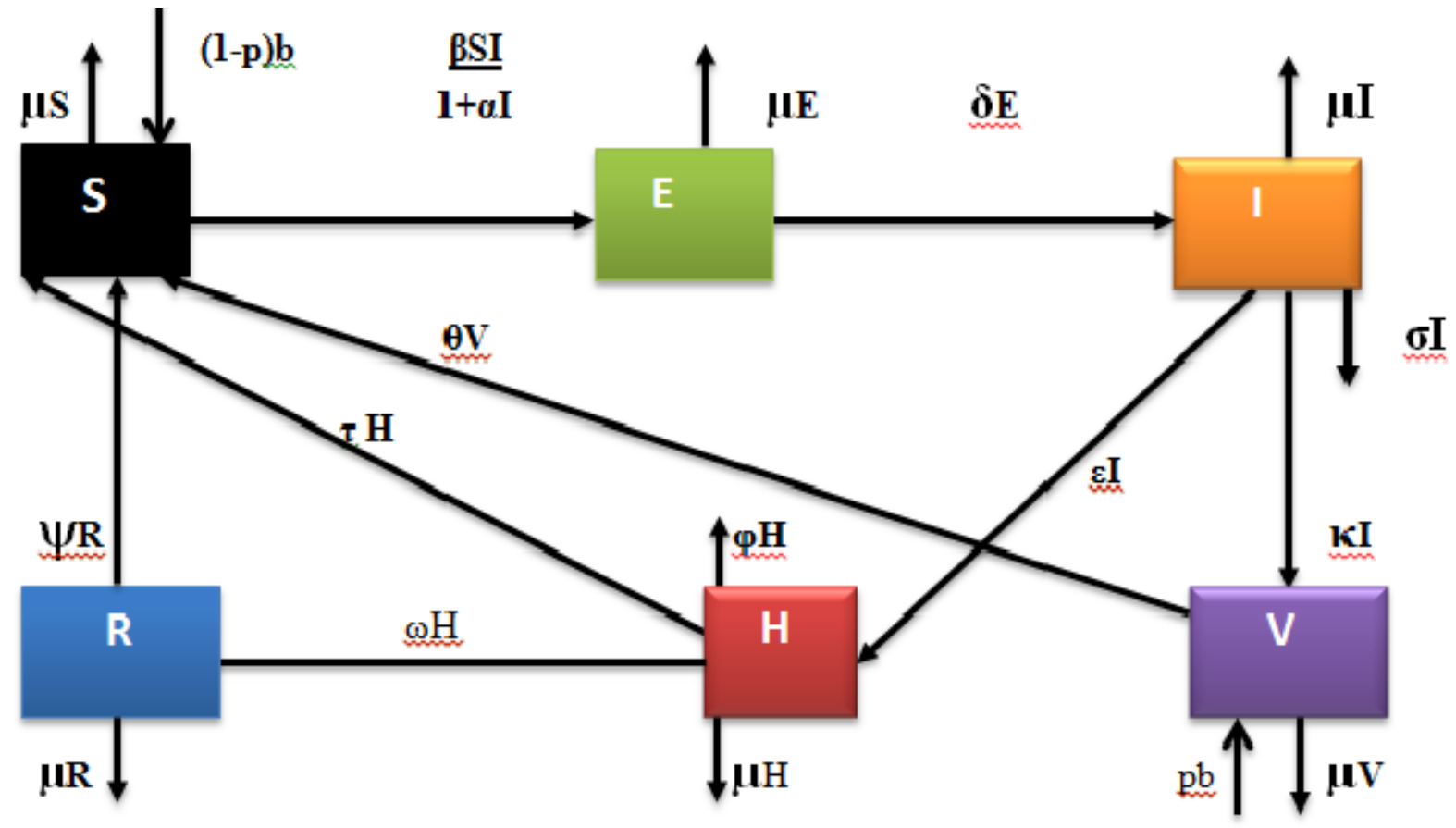

Figure 1: Flow chart.

$$
\begin{aligned}
\frac{d S(t)}{d t} & =(1-\mathrm{p}) \mathrm{b}-\frac{\beta S(t) I(t)}{1+\alpha I}-\mu S(t)+\theta V(t)+\psi R(t)+\tau H, \\
\frac{d E(t)}{d t} & =\frac{\beta S(t) I(t)}{1+\alpha I}-(\mu+\delta) E(t), \\
\frac{d I(t)}{d t} & =\delta E(t)-(\mu+\sigma+\varepsilon+\kappa) I(t), \\
\frac{d V(t)}{d t} & =p b+\kappa I-\mu V(t)-\theta V(t), \\
\frac{d H(t)}{d t} & =\varepsilon I-\tau H-\mu H-\omega H-\varphi H(t), \\
\frac{d R(t)}{d t} & =\omega H(t)-\mu R(t)-\psi R(t),
\end{aligned}
$$

Subject to initial conditions

$$
\begin{aligned}
& S(0)=S_{0} \geq 0, E(0)=E_{0} \geq 0, I(0)=I_{0} \geq 0, \quad V(0)=V_{0} \geq 0, \\
& H(0)=H_{0} \geq 0, R(0)=R_{0} \geq 0 .
\end{aligned}
$$


Here, in model (1), the population growth rate is shown by b. The disease contact rate for the susceptible individuals is represented by $\beta, \mu$ is the natural death rate for the population, $\theta$ the vaccine waning rate and at the rate of $\psi$ the individuals become susceptible again. The exposed individuals are become infected at the rate of $\delta$ and move to the infected class. At $\kappa$ rate the infected individuals are treated $\mathrm{p}$ is the proportion of individuals that's vaccinated. The number of people recovered from infection is represented by $\omega$. The infected individual dies due to disease at the rate of $\sigma$ and $\alpha$ shows the effect of saturated incidence. $\tau$ is rate of flow from hospitalize to susceptible and $\epsilon$ is the rate at which infected individuals are hospitalized. The individual's death rate in hospitalized class is denoted by $\varphi$.

Total dynamics of the population is given by

$\frac{d N}{d t}=\mathrm{b}-\mu N-\sigma I-\phi H$

$\frac{d N}{d t} \leq \mathrm{b}-\mu N$

$\lim _{t \rightarrow \infty} \sup N(t) \leq \frac{b}{\mu}$.

2.1 Equilibrium points : In this section, we study the basic properties of the model (1). First, we find the disease free equilibrium, and then, we find the basic reproduction number $R_{o}$. For the disease free equilibrium, we equate the left hand side of the system (1), to zero about the point $E^{0}=\left(S^{0}, 0,0, V^{0}, 0,0\right)$, we obtain

$$
S^{0}=(1-\mathrm{p}) N^{0}+N^{0} \frac{\theta \mathrm{p}}{(\mu+\theta)} \quad \text { and } \quad V^{0}=\frac{b \mathrm{p}}{(\mu+\theta)}
$$

Where

$$
N^{0}=\frac{b}{\mu}
$$

2.2 Basic reproduction number: The basic reproduction number for the system (1) is obtaining by using the method of [13]. The basic reproduction number represents an average number of secondary infection generates when a single infection is introduced into a purely susceptible population, the rest is assumed to be susceptible.

$$
\begin{gathered}
\text { Let } X=(E, I) \\
\frac{d x}{d t}=\mathcal{F}-v \\
\mathcal{F}=\left[\begin{array}{c}
\frac{\beta S I}{1+\alpha I} \\
O
\end{array}\right] \\
v=\left[\begin{array}{c}
(\delta+\mu) E \\
-\delta E+(\mu+\sigma+\varepsilon+\kappa) I
\end{array}\right] \\
\mathrm{F}=\text { Jacobean of } \mathcal{F} \text { at DFE }=\left[\begin{array}{cc}
0 & \beta S^{0} \\
0 & 0
\end{array}\right] \\
\mathrm{V}=\text { Jacobean of } v \text { at DFE }=\left[\begin{array}{cc}
(\mu+\delta) & 0 \\
-\delta & (\mu+\sigma+\varepsilon+\kappa)
\end{array}\right]
\end{gathered}
$$

The product of $F V^{-1}$

$R_{0}=\frac{1}{(\mu+\delta)(\mu+\sigma+\varepsilon+\kappa)}\left[\begin{array}{cc}0 & \beta S^{0} \\ 0 & 0\end{array}\right]\left[\begin{array}{cc}(\mu+\sigma+\varepsilon+\kappa) & 0 \\ \delta & (\mu+\delta)\end{array}\right]$ 


$$
R_{0}=\frac{\beta \delta S^{0}}{(\mu+\delta)(\mu+\sigma+\varepsilon+\kappa)}
$$

$R_{o}$ is the required basic reproduction number for system (1). In case of disease free, the basic reproduction number $R_{0}<1$, shows that DFE is stable, and the disease may be controlled via some effective vaccination and prevention. In case when the threshold quantity exceeds unity the disease exists in the community that's become endemic.

\subsection{Endemic equilibrium}

For endemic equilibria, setting left side of the system (1), equating zero, about the endemic equilibrium point $E^{1}=$ $\left(S^{*}, E^{*}, I^{*}, V^{*}, H^{*}, R^{*}\right)$, we obtain,

$$
\begin{aligned}
S^{*} & =\frac{(\mu+\delta)(\mu+\sigma+\varepsilon+\kappa)\left(1+\alpha I^{*}\right)}{\beta \delta} \\
E^{*} & =\frac{(\mu+\sigma+\varepsilon+\kappa)}{\delta} I^{*} \\
V^{*} & =\frac{b p+k I^{*}}{(\mu+\theta)} \\
H^{*} & =\frac{\varepsilon I^{*}}{(\tau+\mu+\omega+\varphi)} \\
R^{*} & =\frac{\omega \varepsilon I^{*}}{(\mu+\omega)(\tau+\mu+\omega+\varphi)}
\end{aligned}
$$

3. Stability analysis : In this section, we investigate the stability analysis of the disease free and endemic equilibrium. In subsection 3.1, we find the local stability of the disease free case when the threshold quantity is less than unity. In subsequent section 3.2, we find out the local stability of endemic equilibrium when the threshold quantity exceeds unity.

3.1 Local stability : To understand the behavior of the system (1), we set left hand side of the system (1), equal to zero, about $E^{o}$, we obtain the following Jacobean matrix $J_{o}$.

Theorem 3.1. The disease-free equilibrium (DFE) point $E^{0}$ is stable locally asymptotically, if $R_{o} \leq 1$, otherwise an unstable equilibrium point exists.

Proof: To show the result, we use the system (1), about the disease free equilibrium point $E^{o}$, equal to zero, the left hand side of the system (1), and obtain the following Jacobean matrix $J_{o}$.

$$
J_{0}=\left[\begin{array}{cccccc}
-\gamma & 0 & -\beta S^{0} & \theta & \tau & \psi \\
0 & -(\mu+\delta) & \beta S^{0} & 0 & 0 & 0 \\
0 & \delta & -(\mu+\alpha+\varepsilon+\kappa) & 0 & 0 & 0 \\
0 & 0 & k & -(\mu+\theta) & 0 & 0 \\
0 & 0 & \varepsilon & 0 & (\tau+\mu+\omega+\varphi) & 0 \\
0 & 0 & 0 & 0 & \omega & -(\mu+\psi)
\end{array}\right]
$$

The Jacobean matrix $J_{o}$, reducing to echelon form, we write in the following form: 


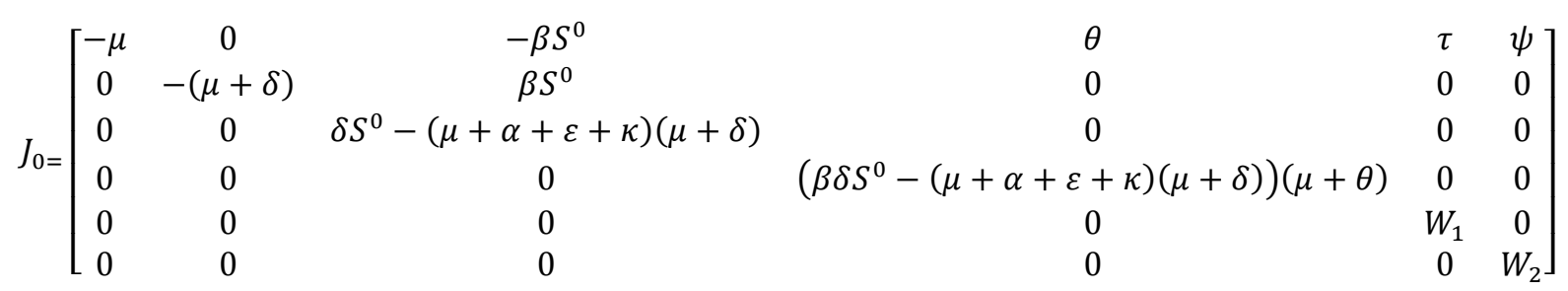

Where,

$$
\begin{aligned}
& W_{1}=\left(\beta \delta S^{0}-(\mu+\alpha+\varepsilon+\kappa)(\mu+\delta)\right)(\mu+\theta)(\tau+\mu+\omega+\varphi), \\
& W_{2}=\left(\beta \delta S^{0}-(\mu+\alpha+\varepsilon+\kappa)(\mu+\delta)\right)(\mu+\theta)(\tau+\mu+\omega+\varphi)(\mu+\psi)
\end{aligned}
$$

The associated characteristics equation of $J_{o}$, is given by:

$(\lambda+\mu)[\lambda+(\mu+\delta)] \quad\left[\lambda-\left\{\beta \delta S^{0}-(\mu+\alpha+\varepsilon+\kappa)(\mu+\delta)\right\}\left[\lambda-\left\{\left(\beta \delta S^{0}-(\mu+\alpha+\varepsilon+\kappa)(\mu+\delta)\right)(\mu+\right.\right.\right.$ $\theta)\}]\left[\lambda-\left\{\left(\beta \delta S^{0}-(\mu+\alpha+\varepsilon+\kappa)(\mu+\delta)\right)(\mu+\theta)(\tau+\mu+\omega+\varphi)\right\}\right]\left[\lambda-\left\{\left(\beta \delta S^{0}-(\mu+\alpha+\varepsilon+\kappa)(\mu+\right.\right.\right.$ $\delta))(\mu+\theta)(\tau+\mu+\omega+\varphi)(\mu+\psi)\}]=0$

A little arrangement, we can write the above equation as:

$(\lambda+\mu)[\lambda+(\mu+\delta)] \quad\left[\lambda-(\mu+\alpha+\varepsilon+\kappa)(\mu+\delta)\left(R_{0}-1\right)\right]\left[\lambda-(\mu+\alpha+\varepsilon+\kappa)(\mu+\delta)(\mu+\theta)\left(R_{0}-1\right)[\lambda-\right.$ $\left.(\mu+\alpha+\varepsilon+\kappa)(\mu+\delta))(\mu+\theta)(\tau+\mu+\omega+\varphi)\left(R_{0}-1\right)\right][\lambda-(\mu+\alpha+\varepsilon+\kappa)(\mu+\delta))(\mu+\theta)(\tau+\mu+\omega+$ $\left.\varphi)(\mu+\psi)\left(R_{0}-1\right)\right]=0$

$\lambda_{1}=-\mu<0$,

$\lambda_{2}=-(\mu+\delta)<0$,

$\lambda_{3}=(\mu+\alpha+\varepsilon+\kappa)(\mu+\delta)\left(R_{0}-1\right)$

$\lambda_{4}=(\mu+\alpha+\varepsilon+\kappa)(\mu+\delta)(\mu+\theta)\left(R_{0}-1\right)<0$,

$\left.\lambda_{5}=(\mu+\alpha+\varepsilon+\kappa)(\mu+\delta)\right)(\mu+\theta)(\tau+\mu+\omega+\varphi)\left(R_{0}-1\right)<0$.

$\left.\lambda_{6}=(\mu+\alpha+\varepsilon+\kappa)(\mu+\delta)\right)(\mu+\theta)(\tau+\mu+\omega+\varphi)(\mu+\psi)\left(R_{0}-1\right)<0$.

The first two eigenvalues are clearly negative, and the rest of four eigenvalues depends upon $R_{0}$. If $R_{0}<1$, the rest of the eigenvalues are negative. Thus, all the eigenvalues of Jacobean matrix $J_{o}$, are negative if and only if $R_{0}<$ 1 , the disease free equilibrium point $E^{o}$, is locally asymptotically stable. So, by Routh-Hurtwiz criteria the system (1), about $E^{o}$, is locally asymptotically stable. Next, we show the local stability of the endemic equilibrium point $E^{1}$.

Theorem 3.2: For $R_{0}>1$, the endemic equilibrium $E^{1}$ is stable locally asymptotically and unstable otherwise.

Proof: The Jacobean matrix $J_{1}$ about the endemic equilibrium point $E^{1}$, is given by

$$
J_{1}=\left[\begin{array}{cccccc}
-\left(\mu+\frac{\beta I}{1+\alpha I}\right) & 0 & -\frac{\beta S}{(1+\alpha I)^{2}} & \theta & \tau & \psi \\
\frac{\beta S I}{1+\alpha I} & -(\mu+\delta) & \frac{\beta S}{(1+\alpha I)^{2}} & 0 & 0 & 0 \\
0 & \delta & -(\mu+\alpha+\varepsilon+\kappa) & 0 & 0 & 0 \\
0 & 0 & k & -(\mu+\theta) & 0 & 0 \\
0 & 0 & \varepsilon & 0 & (\tau+\mu+\omega+\varphi) & 0 \\
0 & 0 & 0 & 0 & \omega & -(\mu+\psi)
\end{array}\right]
$$




$$
J_{1}=\left[\begin{array}{cccccc}
-\left(\mu+\frac{\beta I}{1+\alpha I}\right) & 0 & -\frac{\beta S}{(1+\alpha I)^{2}} & \theta & \tau & \psi \\
o & -\left(\mu+\frac{\beta I}{1+\alpha I}\right)(\mu+\delta) & \frac{\mu \beta S}{(1+\alpha I)^{2}} & \frac{\theta \beta I}{1+\alpha I} & \frac{\tau \beta I}{1+\alpha I} & \frac{\psi \beta I}{1+\alpha I} \\
0 & 0 & M_{1} & \frac{\delta \theta \beta I}{1+\alpha I} & \frac{\delta \tau \beta I}{1+\alpha I} & \frac{\delta \psi \beta I}{1+\alpha I} \\
0 & 0 & 0 & M_{2} & \frac{k \delta \tau \beta I}{1+\alpha I} & \frac{k \delta \psi \beta I}{1+\alpha I} \\
0 & 0 & 0 & 0 & M_{3} & M_{4} \\
0 & 0 & 0 & 0 & 0 & M_{5}
\end{array}\right]
$$

Where $M_{1}=-\left(\mu+\frac{\beta I}{1+\alpha I}\right)(\mu+\delta)(\mu+\alpha+\varepsilon+\kappa)+\frac{\delta \mu \beta S}{(1+\alpha I)^{2}}$

$M_{2}=(\mu+\theta) M_{1}+\frac{k \delta \theta \beta I}{1+\alpha I}$

$M_{3}=M_{2}\left(\frac{\varepsilon \delta \tau \beta I}{1+\alpha I}\right)-\left(\frac{\epsilon \delta \theta \beta I}{1+\alpha I}\right)\left(\frac{k \delta \tau \beta I}{1+\alpha I}\right)$

$M_{4}=M_{2} \frac{\epsilon \delta \psi \beta I}{1+\alpha I}-\left(\frac{k \delta \psi \beta I}{1+\alpha I}\right)\left(\frac{\epsilon \delta \theta \beta I}{1+\alpha I}\right)$

The characteristic equation becomes,

$\left(\lambda+\left(\mu+\frac{\beta I}{1+\alpha I}\right)\right)\left(\lambda+\left(\mu+\frac{\beta I}{1+\alpha I}\right)(\mu+\delta)\right)\left(\lambda-M_{1}\right)\left(\lambda-M_{2}\right)\left(\lambda-M_{3}\right)\left(\lambda-M_{5}\right)=0$

After simplification, we get the eigenvalues as given below,

$\lambda_{1}=-\left(\mu+\frac{\beta I}{1+\alpha I}\right)<0$

$\lambda_{2}=-\left(\mu+\frac{\beta I}{1+\alpha I}\right)(\mu+\delta)<0$

$\lambda_{3}=M_{1}=-(\mu+\delta)(\mu+\alpha+\varepsilon+\kappa)\left(\frac{\beta I+\mu \alpha I}{1+\alpha I}\right)<0$

$\lambda_{4}=-\frac{[(\mu+\delta)(\mu+\theta)(\mu+\alpha+\varepsilon+\kappa) \mu \alpha I+(\mu+\delta)(\mu+\theta)(\mu+\alpha+\varepsilon) \beta I+(\mu+\delta+\theta) \mu k]}{1+\alpha I}<0$

$\lambda_{5}=M_{3}=\lambda_{4}\left(\frac{\varepsilon \delta \tau \beta I}{1+\alpha I}\right)-\left(\frac{\epsilon \delta \theta \beta I}{1+\alpha I}\right)\left(\frac{k \delta \tau \beta I}{1+\alpha I}\right)<0$ As $\lambda_{4}<0$

$\lambda_{6}=M_{5}=(\mu+\psi) \lambda_{5}+\omega\left[\lambda_{4}\left(\frac{\epsilon \delta \psi \beta I}{1+\alpha I}\right)-\left(\frac{k \delta \psi \beta I}{1+\alpha I}\right)\left(\frac{\epsilon \delta \theta \beta I}{1+\alpha I}\right)\right]<0$ As $\lambda_{4}<0, \lambda_{5}<0$

So, the eigenvalues associated with system (1) have negative real parts. Therefore, the system (1) is locally asymptotically stable about the endemic equilibrium point $E^{1}$. 
4. Global Stability Analysis: In this section, we discuss the global stability of disease free and endemic equilibrium. First, we show the global stability of the disease free equilibrium of the system (1) about the equilibrium point $E^{o}$. Here, $\mathrm{N}(\mathrm{t})$ the total population which satisfies the equation, $\frac{d N}{d t} \leq \mathrm{b}-\mu N, N \rightarrow \frac{\mathrm{b}}{\mu}$, as $t \rightarrow \infty$. The analytic result are can be obtain for system (1), by which we assumed the population to be $\operatorname{constant} \mathrm{N}=N^{0}=\frac{\mathrm{b}}{\mu}$, see $[14,15]$. The reduced dynamical system is presented in the following:

$$
\begin{aligned}
& \frac{d s}{d t}=b(1-p)+\frac{\theta b p}{\mu+\theta}-\frac{\beta S(t) I(t)}{1+\alpha I}-\mu S(t)+\left(\frac{\theta k}{\mu+\theta}+\frac{\tau \varepsilon}{\mu+\epsilon+\tau+\phi}\right) I(t) \\
& \frac{d E(t)}{d t}=\frac{\beta S(t) I(t)}{1+\alpha I}-(\mu+\delta) E(t) \\
& \frac{d I(t)}{d t}=\delta E(t)-(\mu+\sigma+\varepsilon+\kappa) I(t)
\end{aligned}
$$

4.1 Global stability of disease free equilibrium:

In this subsection, we show the global stability of the disease free equilibrium $E^{o}$. To show this result, we use the method used and developed by Castillo-Chavez and his co-authors [14]. In the following we present the two conditions, which guarantee the global stability of the disease-free state.

$$
\begin{aligned}
& \frac{d X}{d t}=F(X, Z), \\
& \frac{d Z}{d t}=G(X, Z), G(X, O)=0,
\end{aligned}
$$

Where $\quad \mathrm{X}=(\mathrm{S}), \quad \mathrm{Z}=(\mathrm{E}, \mathrm{I})$; with $\mathrm{X} \in R \& Z \in R^{2}$

$\mathrm{X}$ shows the number of uninfected individuals and $\mathrm{Z}$ represents the number of infected individuals. The following two conditions (H1) and (H2) must be met to guarantee a local asymptotic stability

H1: $\quad$ For $\frac{d X}{d t}=F(X, 0), X^{0}$ is globally asymptotically stable.

H2: $\quad \mathrm{G}(\mathrm{X}, \mathrm{Z})=\mathrm{BZ}-\widehat{G}(\mathrm{X}, \mathrm{Z})$ where $\widehat{G}(\mathrm{X}, \mathrm{Z}) \geq 0$ for $(\mathrm{X}, \mathrm{Z}) \in \Omega$.

Where $\mathrm{B}$ is an M-matrix, all their entries in off diagonal are non-negative. The symbol $\Omega$ is the biologically feasible region.

Theorem 4.1: Suppose $R_{0}<1$, the disease free equilibrium $E^{o}$ is globally asymptotically stable.

Proof: let $\mathrm{X}=(\mathrm{S})$, and $\mathrm{Z}=(\mathrm{E}, \mathrm{I})$ and

$Q_{0}=\left(X^{0}, 0\right)$ where $S^{0}=X^{0}=\frac{\mathrm{b}}{\mu}(1-p)+\frac{\theta}{\mu}\left(\frac{p b}{\mu+\theta}\right)$

Then,

$\frac{d X}{d t}=b(1-p)+\frac{\theta b p}{\mu+\theta}-\frac{\beta S(t) I(t)}{1+\alpha I}-\mu S(t)+\left(\frac{\theta k}{\mu+\theta}+\frac{\tau \varepsilon}{\mu+\epsilon+\tau+\phi}\right) I(t)$

When $S=S^{o}$,

$\frac{d X}{d t}=F(X, 0)=. b(1-p)+\frac{\theta b p}{\mu+\theta}-\mu S(t)$

$$
F(X, 0)=0
$$

and

Or 
$\frac{d X^{0}}{d t}=(1-p)+\frac{\theta b p}{\mu+\theta}-\mu S^{0}$

$\frac{d X^{0}}{d t}=(1-p)+\frac{\theta b p}{\mu+\theta}-\mu\left[\frac{\mathrm{b}}{\mu}(1-p)+\frac{\theta}{\mu}\left(\frac{p b}{\mu+\theta}\right)\right]$

Thus, $X^{0}$ is globally asymptotically stable.

In the reduced system (3), we obtain the following form:

$$
\mathrm{G}(\mathrm{X}, \mathrm{Z})=\left[\begin{array}{cc}
-(\mu+\delta) & \beta S^{0} \\
\delta & -(\mu+\sigma+\epsilon+k)
\end{array}\right]\left[\begin{array}{l}
E \\
I
\end{array}\right]-\left[\begin{array}{c}
\beta S^{0} I-\frac{\beta S I}{1+\alpha I} \\
0
\end{array}\right]=\mathrm{BZ}-\widehat{G}(\mathrm{X}, \mathrm{Z}) .
$$

Where

$\mathrm{B}=\left[\begin{array}{cc}-(\mu+\delta) & \beta S^{0} \\ \delta & -(\mu+\sigma+\epsilon+k)\end{array}\right]$, and $\widehat{G}(\mathrm{X}, \mathrm{Z})=\left[\begin{array}{c}\beta S^{0} I-\frac{\beta S I}{1+b I} \\ 0\end{array}\right]$

$\widehat{G}(\mathrm{X}, \mathrm{Z}) \geq 0$, and clearly B is an M-matrix, thus (H1) and (H2) are satisfied.

Hence, the disease free equilibrium about the point $E^{0}$ is globally asymptotically stable.

Theorem: 4.2.1: If $R_{0}>1$, the endemic equilibrium $E^{1}$ of the reduced system (3), is globally asymptotically stable, unstable for $R_{o} \leq 1$.

Proof: To prove this result, the Jacobean matrix $J^{*}$, for the reduced system (3), is given in the following.

$$
J^{*}=\left[\begin{array}{ccc}
-\left(\mu+\frac{\beta I}{1+b I}\right) & 0 & \frac{\theta k}{\mu+\theta}+\frac{\tau \varepsilon}{\mu+\omega+\tau+\phi}-\frac{\beta S}{(1+b I)^{2}} \\
\frac{\beta I}{1+b I} & -(\mu+\delta) & \frac{\beta S}{(1+b I)^{2}} \\
0 & \delta & -(\mu+\sigma+\epsilon+k)
\end{array}\right]
$$

Second additive compound matrix, for $3 \times 3$ matrix is given by:

$$
J^{[2]}=\left[\begin{array}{ccc}
a_{11}+a_{22} & a_{23} & -a_{13} \\
a_{32} & a_{11}+a_{33} & a_{12} \\
a_{31} & a_{21} & a_{22}+a_{33}
\end{array}\right]
$$

We obtain the second additive compound matrix for our system (3) as:

$$
J^{[2]}=\left[\begin{array}{ccc}
-\left(2 \mu+\delta+\frac{\beta I}{1+b I}\right) & \frac{\beta S}{(1+b I)^{2}} & \frac{\beta S}{(1+b I)^{2}}-\frac{\theta k}{\mu+\theta}-\frac{\tau \varepsilon}{\mu+\omega+\tau+\phi} \\
\delta & -\left(2 \mu+\sigma+\epsilon+k+\frac{\beta I}{1+b I}\right) & 0 \\
0 & \frac{\beta I}{1+b I} & -(2 \mu+\delta+\sigma+\epsilon+k)
\end{array}\right]
$$


We consider the function, $\quad \mathrm{P}=\mathrm{P}(\mathrm{S}, \mathrm{E}, \mathrm{I})=\left(\begin{array}{ccc}1 & 0 & 0 \\ 0 & \frac{E}{I} & 0 \\ 0 & 0 & \frac{E}{I}\end{array}\right)$,

$$
P^{-1}=\left(\begin{array}{ccc}
1 & 0 & 0 \\
0 & \frac{I}{E} & 0 \\
0 & 0 & \frac{I}{E}
\end{array}\right)
$$

$P_{f}=\left(\begin{array}{ccc}0 & 0 & 0 \\ 0 & \frac{\dot{E}}{I}-\frac{E \dot{I}}{I^{2}} & 0 \\ 0 & 0 & \frac{\dot{E}}{I}-\frac{E \dot{I}}{I^{2}}\end{array}\right)$,

$P_{f} P^{-1}=\left(\begin{array}{ccc}0 & 0 & 0 \\ 0 & \frac{\dot{E}}{E}-\frac{\dot{I}}{I} & 0 \\ 0 & 0 & \frac{\dot{E}}{E}-\frac{\dot{I}}{I}\end{array}\right)$

$\mathrm{P} J^{[2]} P^{-1}=\left[\begin{array}{ccc}-\left(2 \mu+\delta+\frac{\beta I}{1+b I}\right) & \frac{\beta S}{(1+b I)^{2}} \frac{I}{E} & \frac{I}{E}\left(\frac{\beta S}{(1+b I)^{2}}-\frac{\theta k}{\mu+\theta}-\frac{\tau \varepsilon}{\mu+\omega+\tau+\phi}\right) \\ \frac{E}{I} \delta & -\left(2 \mu+\sigma+\epsilon+k+\frac{\beta I}{1+b I}\right) & 0 \\ 0 & \frac{\beta I}{1+b I} & -(2 \mu+\delta+\sigma+\epsilon+k)\end{array}\right]$

$\mathrm{B}=P_{f} P^{-1}+\mathrm{P} J^{[2]} P^{-1}$

$\Rightarrow\left[\begin{array}{ccc}-\left(2 \mu+\delta+\frac{\beta I}{1+b I}\right) & \frac{\beta S}{(1+b I)^{2}} \frac{I}{E} & \frac{I}{E}\left(\frac{\beta S}{(1+b I)^{2}}-\frac{\theta k}{\mu+\theta}-\frac{\tau \varepsilon}{\mu+\omega+\tau+\phi}\right) \\ \frac{E}{I} \delta & \left(\frac{\dot{E}}{E}-\frac{I}{I}\right)-\left(2 \mu+\sigma+\epsilon+k+\frac{\beta I}{1+b I}\right) & 0 \\ 0 & \frac{\beta I}{1+b I} & \left(\frac{\dot{E}}{E}-\frac{I}{I}\right)-(2 \mu+\delta+\sigma+\epsilon+k)\end{array}\right]$

$\mathrm{B}=\left(\begin{array}{ll}B_{11} & B_{12} \\ B_{21} & B_{22}\end{array}\right)$

$B_{11}=-\left(2 \mu+\delta+\frac{\beta I}{1+b I}\right)$

$B_{12}=\left[\frac{\beta S}{(1+b I)^{2}} \frac{I}{E}, \quad \frac{I}{E}\left(\frac{\beta S}{(1+b I)^{2}}-\frac{\theta k}{\mu+\theta}-\frac{\tau \varepsilon}{\mu+\omega+\tau+\phi}\right)\right]$ 


$$
\begin{aligned}
& B_{21}=\left[\begin{array}{c}
\frac{E}{I} \delta \\
0
\end{array}\right] \\
& B_{22}=\left[\begin{array}{cc}
\left(\frac{\dot{E}}{E}-\frac{\dot{I}}{I}\right)-\left(2 \mu+\sigma+\epsilon+k+\frac{\beta I}{1+b I}\right) & 0 \\
\frac{\beta I}{1+b I} & \left(\frac{\dot{E}}{E}-\frac{\dot{I}}{I}\right)-(2 \mu+\delta+\sigma+\epsilon+k)
\end{array}\right]
\end{aligned}
$$

Now

$\mu(B) \leq \sup \left(g_{1}, g_{2}\right)=\sup \left\{\mu_{1}\left(B_{(11)}+\left|B_{12}\right|\right), \mu_{1}\left(B_{(22)}+\left|B_{21}\right|\right)\right\}$

$g_{1}=\mu_{1}\left(B_{11}+\left|B_{12}\right|\right)$

$g_{1}=-\left(2 \mu+\delta+\frac{\beta I}{1+\alpha I}\right)+\frac{\beta S}{(1+\alpha I)^{2}} \cdot \frac{I}{E}$

$\leq-\left(2 \mu+\delta+\frac{\beta I}{1+\alpha I}\right)+\frac{\beta S}{1+\alpha I} \cdot \frac{I}{E}$ using second equation of reduced system (3),

$\leq \frac{\dot{E}}{E}-\mu$

$g_{2}=\mu_{1}\left(B_{(22)}+\left|B_{21}\right|\right)$

$\leq\left(\frac{\dot{E}}{E}-\frac{\dot{I}}{I}\right)-(2 \mu+\delta+\sigma+\epsilon+k)+\frac{E}{I} \delta$

$\leq \frac{\dot{E}}{E}-\frac{\dot{I}}{I}-\left(2 \gamma+\gamma_{1}+\alpha+\mu\right)+\frac{E}{I} \delta$, using third equation of reduced system (3),

$\leq \frac{\dot{E}}{E}-\mu$

$\mu(B) \leq \sup \left(g_{1}, g_{2}\right) \leq \frac{\dot{E}}{E}-\mu$

$\mu(B) \leq \frac{\dot{E}}{E}-\mu$

$\mathrm{q}=\frac{1}{t} \int_{0}^{t} \mu(B) d s \leq \frac{1}{t} \int_{0}^{t}\left(\frac{\dot{E}}{E}-\mu\right) d s=\frac{1}{t} \log \frac{E(t)}{E(0)}-\mu=-\frac{\mu}{2}<0$

Hence,

$$
\mathrm{q} \leq-\frac{\mu}{2}
$$

Implies that, $\quad q=-\frac{\mu}{2}<0$. Thus, the result [16] implies that the positive equilibrium point of $E^{1}$ is globally asymptotically stable. 
5. Discussion: We have successfully presented Stability analysis of SEIVHR epidemic model with saturated incidence rate. Initially, the formulation of the problem and their basic properties has been presented. The basic reproduction number $R_{0}$ for the model is derived. We observed that the disease free equilibrium is stable locally and globally when $R_{0}<1$. Unstable equilibrium of the system at disease free equilibrium exists when $R_{0}>1$. Further, we proved that the local stability of endemic equilibrium exits and locally asymptotically stable when $R_{0}>1$. We obtained the global stability of an endemic equilibrium by using the geometric approach. It is concluded when the reproduction number exceeds unity the global stability for the system exists. Some numerical results have been presented for the justification of theoretical results. Figure 2, represents the behavior of individuals for disease Free State. Figure 3, shows the behavior of individuals for the endemic state. The parameter values in numerical simulations are presented in Table 1.

Table 1. Estimated Values.

\begin{tabular}{|c|l|l|}
\hline Parameter & Description & Value \\
\hline $\boldsymbol{\beta}$ & The population growth rate & 1.9 \\
$\boldsymbol{\mu}$ & The disease contact rate & 0.56 \\
$\boldsymbol{\theta}$ & The natural death rate for the population & 0.02 \\
$\boldsymbol{\psi}$ & The vaccine waning rate & 0.4 \\
$\boldsymbol{\delta}$ & The rate at which individuals become susceptible again & 0.056 \\
$\mathbf{k}$ & The exposed individuals are become infected & 0.3 \\
$\mathbf{p}$ & Rate the infected individuals are treated & 0.1 \\
$\boldsymbol{\omega}$ & The proportion of individuals that's vaccinated & 0.3 \\
$\boldsymbol{\sigma}$ & The number of people recovered from infection & 0.2 \\
$\boldsymbol{\alpha}$ & Disease death rate & 0.03 \\
$\boldsymbol{\tau}$ & Effect of saturated incidence & 2 \\
$\boldsymbol{\epsilon}$ & Rate of flow from H to S & The rate at which invectives are hospitalized \\
$\boldsymbol{\varphi}$ & Disease rate of hospitalized people & 0.02 \\
\hline
\end{tabular}




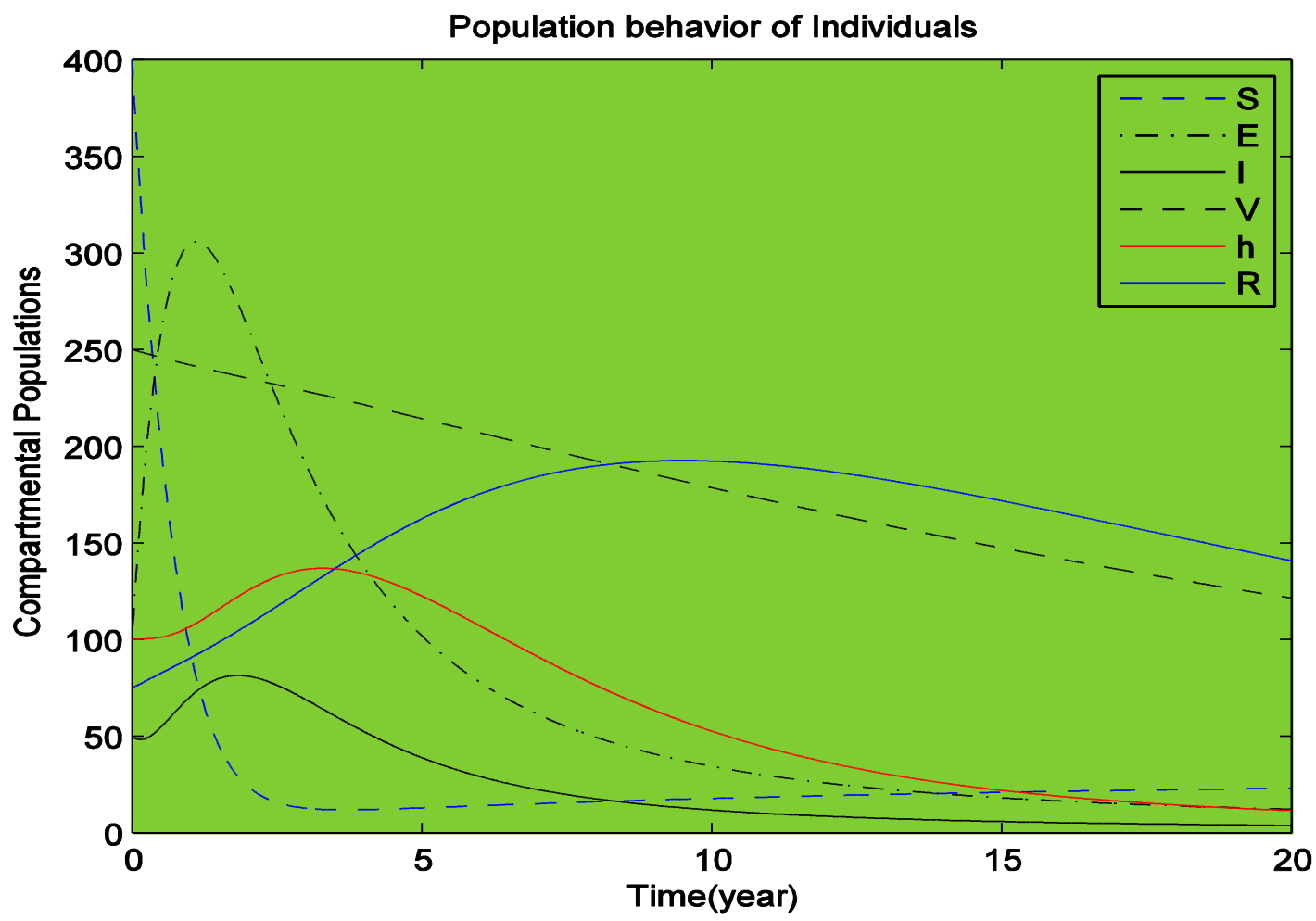

Figure 2: Population behavior of individuals at disease free case.

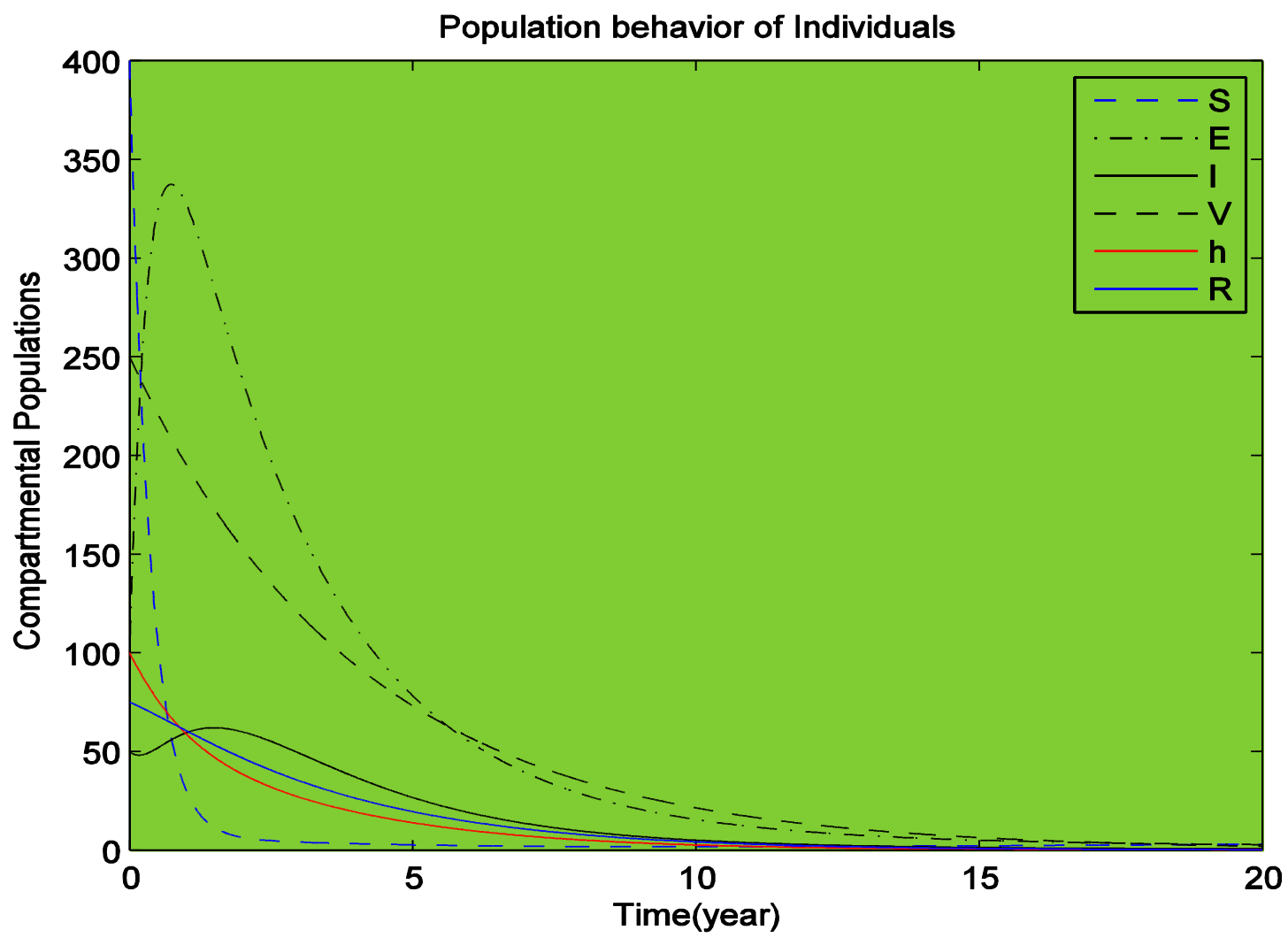

Figure 3: Population behavior of individuals at endemic equilibrium point. 


\section{REFERENCES}

[1] Kermack, W. O., \& McKendrick, A. G. (1927, August). A contribution to the mathematical theory of epidemics. In Proceedings of the Royal Society of London A: Mathematical, Physical and Engineering Sciences (Vol. 115, No. 772, pp. 700-721). The Royal Society.

[2] Anderson, R. M., \& May, R. M. (1991). Infectious diseases of humans (Vol. 1). Oxford: Oxford university press.

[3] Zhou, X., \& Cui, J. (2011). Analysis of stability and bifurcation for an SEIV epidemic model with vaccination and nonlinear incidence rate. Nonlinear Dynamics, 63(4), 639-653.

[4] Liu, W. M., Hethcote, H. W., \& Levin, S. A. (1987). Dynamical behavior of epidemiological models with nonlinear incidence rates. Journal of mathematical biology, 25(4), 359-380.

[5] Alexander, M. E., \& Moghadas, S. M. (2005). Bifurcation analysis of an SIRS epidemic model with generalized incidence. SIAM Journal on Applied Mathematics, 65(5), 1794-1816.

[6] Li, M., \& Liu, X. (2014, February). An SIR epidemic model with time delay and general nonlinear incidence rate. In Abstract and Applied Analysis (Vol. 2014). Hindawi Publishing Corporation.

[7] Wang, J. J., Zhang, J. Z., \& Jin, Z. (2010). Analysis of an SIR model with bilinear incidence rate. Nonlinear Analysis: Real World Applications, 11(4), 2390-2402.

[8] Xu, R., \& Ma, Z. (2009). Stability of a delayed SIRS epidemic model with a nonlinear incidence rate. Chaos, Solitons \& Fractals, 41(5), 2319-2325.

[9] Korobeinikov, A. (2007). Global properties of infectious disease models with nonlinear incidence. Bulletin of Mathematical Biology, 69(6), 1871-1886.

[10] Fiore, A. E., Bridges, C. B., \& Cox, N. J. (2009). Seasonal influenza vaccines. In Vaccines for Pandemic Influenza (pp. 43-82). Springer Berlin Heidelberg.

[11] Chang, Y., Brewer, N. T., Rinas, A. C., Schmitt, K., \& Smith, J. S. (2009). Evaluating the impact of human papillomavirus vaccines. Vaccine, 27(32), 4355-4362.

[12] Liesegang, T. J. (2009). Varicella zoster virus vaccines: effective, but concerns linger. Canadian Journal of Ophthalmology/Journal Canadien d'Ophtalmologie, 44(4), 379-384.

[13] Van den Driessche, P., \& Watmough, J. (2002). Reproduction numbers and sub-threshold endemic equilibria for compartmental models of disease transmission. Mathematical biosciences, 180(1), 29-48.

[14] Li, M. Y., \& Muldowney, J. S. (1996). A geometric approach to global-stability problems. SIAM Journal on Mathematical Analysis, 27(4), 1070-1083.

[15] Castillo-Chavez, C., \& Yakubu, A. A. (2002). Mathematical Approaches for Emerging and Reemerging Infectious Diseases Part II: Models, Methods, and Theory.

[16] Li, M. Y., \& Muldowney, J. S. (1996). A geometric approach to global-stability problems. SIAM Journal on Mathematical Analysis, 27(4), 1070-1083. 